\title{
Site-specific atomic order and band structure tailoring in the diluted magnetic semiconductor (In,Ga,Mn)As
}

\author{
K. Medjanik, ${ }^{1}$ O. Fedchenko, ${ }^{1}$ O. Yastrubchak, ${ }^{2,3}{ }^{3}$. Sadowski, ${ }^{4,5}$ M. Sawicki, ${ }^{4}$ L. Gluba ${ }^{6},{ }^{6,7}$ D. Vasilyev,${ }^{1}$ S. Babenkov,${ }^{1}$ \\ S. Chernov, ${ }^{1}$ A. Winkelmann $\odot{ }^{8}{ }^{8}$ H. J. Elmers $\odot,{ }^{1}$ and G. Schönhense ${ }^{1}$ \\ ${ }^{1}$ Johannes Gutenberg-Universität, Institut für Physik, D-55128 Mainz, Germany \\ ${ }^{2}$ V. E. Lashkaryov Institute of Semiconductor Physics, National Academy of Sciences of Ukraine, U-03028 Kyiv, Ukraine \\ ${ }^{3}$ Ternopil Ivan Puluj National Technical University, Software Engineering Department, 56, Ruska Street, U-46001 Ternopil, Ukraine \\ ${ }^{4}$ Institute of Physics, Polish Academy of Sciences, Aleja Lotnikow 32/46, PL-02668 Warsaw, Poland \\ ${ }^{5}$ Department of Physics and Electrical Engineering, Linnaeus University, SE-391 82 Kalmar, Sweden \\ ${ }^{6}$ Institute of Agrophysics, Polish Academy of Sciences, Doświadczalna 4, PL-20-290 Lublin, Poland \\ ${ }^{7}$ Institute of Physics, Maria Curie-Sklodowska University in Lublin, Pl. M. Curie-Sktodowskiej 1, PL-20-031 Lublin, Poland \\ ${ }^{8}$ Academic Centre for Materials and Nanotechnology, AGH University of Science and Technology, Mickiewicza 30, PL-30-059 Krakow, Poland
}

(Received 24 August 2020; revised 24 December 2020; accepted 12 January 2021; published 2 February 2021)

Diluted ferromagnetic semiconductors combining ferromagnetic and semiconducting properties in one material provide numerous new functionalities, attractive for basic studies and potentially useful for novel device applications. The tailoring of the electronic structure in analogy to conventional semiconductors has yet to be explored. Here, we demonstrate the conservation of broken inversion symmetry and band structure tailoring for high-quality molecular-beam-epitaxy-grown (In,Ga,Mn)As films with $3 \%$ In plus $2.5 \%$ or $5.6 \%$ Mn using hard-x-ray photoelectron diffraction (hXPD) and momentum microscopy. Photon energies of 3-5 keV ensure that the results are not corrupted by surface effects, which are known to be strong in semiconductors. The missing inversion center of the GaAs host lattice leads to fingerprint-like hXPD signatures of As and Ga sites. For both concentrations, Mn predominantly occupies Ga substitutional sites. Momentum microscopy reveals a shift of the chemical potential with increasing Mn doping and a highly dispersing band, crossing the Fermi level for high Mn concentration. The Mn doping induces a pronounced modification of the spin-orbit split-off band.

DOI: 10.1103/PhysRevB.103.075107

\section{INTRODUCTION}

Diluted ferromagnetic semiconductors (DFSs) have attracted persistent research interest over the past two decades due to the unique combination of ferromagnetism and semiconducting properties in one material [1]. The prototypical DFS (Ga,Mn)As has a remarkably high Curie temperature (close to $200 \mathrm{~K}$ ) [2-4] and served as a testing ground for a number of spintronic devices exploiting spin orientation of charge carriers. Exploiting the unique options of controlling magnetic properties in DFS by light exposure [5], temperature [6], electrostatic gating [7], pressure [8,9], or doping [10], several types of spintronic devices have been proposed and experimentally realized [11].

So far there is no commonly accepted explanation for the origin of the transition from a paramagnetic semiconducting state with localized charge carriers to the metallic state with delocalized carriers in ferromagnetic ( $\mathrm{Ga}, \mathrm{Mn}) \mathrm{As}$ with increasing Mn content [12-15]. Essentially, two models have been proposed to explain the origin of ferromagnetism: the mean-field Zener model $[16,17]$ based on the hybridization of Mn states with the GaAs host bands, and the impurity band model considering the appearance of localized Mn impurity states above the GaAs valence-band maximum [18-20]. Hard-x-ray angular-resolved photoemission spectroscopy (HARPES) [21] and high-resolution ARPES data presented in Ref. [22] reconcile both pictures proposing the coexistence of the two different mechanisms. Exploring the origin of ferromagnetism and the dopant-related changes of the band structure in highly doped DFS would represent an important advance in the understanding of this material class [23].

In this article, we focus on (In, Ga, Mn)As offering additional degrees of freedom: The ability of the parent compound (Ga,Mn)As to form quaternary $(\mathrm{Ga}, \mathrm{Mn})(\mathrm{Bi}, \mathrm{As})$, (In, Ga,Mn)As, and pentanary (In,Ga,Mn)(Bi,As) compounds with a high structural perfection [24,25] offers a complementary experimental path to pinpoint the origin of ferromagnetism. The multicomponent compounds preserve ferromagnetism with the relatively high Curie temperature of (Ga,Mn)As, while enabling a substantial band structure modification (by a replacement of $\mathrm{As}$ by $\mathrm{Bi}$ ) and creation of local strain (by a replacement of Ga by In). Increasing the In content decreases the band gap of the (In, Ga,Mn)As quaternary semiconductor (from about 1.5 to $0.4 \mathrm{eV}$ for In compositions between $0 \%$ and $100 \%$, respectively) and affects the magnetic easy axis direction [26]. In addition, a range of unusual magnetic effects have been reported for (In, Ga,Mn)As [27-33].

Previous photoemission investigations of DFS mainly focused on surface-sensitive methods, while structural 
characterization involves bulk-sensitive diffraction methods, which are usually, due to Friedel's law, not sensitive to inversion asymmetric structures. In the high-doping regime, the dopant site in the host lattice is discussed controversially, and the influence of the Mn content on the band structure is still an open question. Thus, a key experiment for an improved understanding of ferromagnetism in DFS is to combine structural and electronic characterization methods probing the identical sample volume.

We perform hard-X-ray photoelectron diffraction (hXPD) combined with HARPES for (In,Ga,Mn)As thin films with different Mn concentrations for photon energies up to $5.1 \mathrm{keV}$ $[34,35]$. The high kinetic energy of photoelectrons offers a large information depth. Thus, one avoids surface cleaning with the risk of decomposing the surface area. Furthermore, no corroborations of the photoelectron signal by surface states can occur. hXPD gains information about the geometrical structure of the photoemitting atomic layers, surface reconstruction, and relaxations. In particular, we gain information on the integrity of inversion-asymmetric zinc-blende structure independent of the doping level. Moreover, hXPD confirms that the majority of Mn dopants occupy substitutional Ga sites. HARPES reveals the bulk band structure in the same sampled volume as the structural information. The HARPES results reveal a downward shift of the chemical potential with increasing Mn concentration. A highly dispersing band crosses the Fermi level for high Mn concentration. The split-off band shows significant modifications for high Mn concentration. We discuss implications of the photoemission results on the explanation of ferromagnetism in DFS materials.

\section{EXPERIMENT}

\section{A. Sample preparation}

A series of (In, Ga,Mn)As thin-film samples has been prepared by low-temperature molecular-beam epitaxy (MBE) with an In content of $3 \%$ and a Mn content $x$ ranging from $0[(\mathrm{In}, \mathrm{Ga}) \mathrm{As}$ reference layer] to 5.6\%. 30-nm-thick (In,Ga,Mn)As epitaxial layers have been grown at $\sim 230{ }^{\circ} \mathrm{C}$ on GaAs (001) semi-insulating substrates with a predeposited fully relaxed $0.8-\mu \mathrm{m}$-thick $\operatorname{In}_{0.075} \mathrm{Ga}_{0.925} \mathrm{As}$ buffer layer. The buffer layer removes the compressive strain in the top (In,Ga,Mn)As layer, as revealed by photoreflectance studies of the fundamental optical properties of (Ga,Mn)As epitaxial films [36,37]. Tailoring the substrate temperature depending on the intentional Mn content has enabled us to maximize the concentration of substitutional Mn (i.e., assuming the Ga lattice position in $\mathrm{GaAs}$ ), $\mathrm{Mn}_{\mathrm{sub}}$, incorporated into the InGaAs matrix, and to reduce the $\mathrm{As}_{\mathrm{Ga}}$ (antisites) and $\mathrm{Mn}_{\mathrm{I}}$ defect concentration. The MBE growth has been optimized via the $\mathrm{As}_{2}$ flux, i.e., with an $\mathrm{As}_{2} /(\mathrm{Ga}+\mathrm{Mn})$ flux ratio close to the stoichiometric one, as carefully set during the preceding growth of test/calibration samples. For photoemission studies, the (In, Ga, Mn)As layers have been grown on $p$-type conductive substrates to prevent charging effects. The high degree of perfection of a two-dimensional layer-by-layer growth of (In,Ga,Mn)As has been confirmed by reflection high-energy electron diffraction intensity oscillations, usually observed up to the very end of the growth of 30-nm-thick films [38].

\section{B. Characterization of hole densities and magnetic properties}

Micro-Raman spectroscopy was employed to estimate the hole densities in the (In,Ga,Mn)As films. The micro-Raman measurements were performed using an inVia Reflex Raman microscope (Renishaw) at room temperature with the $514.5 \mathrm{~nm}$ argon ion laser line as an excitation source in backscattering configuration from the (001) surfaces of the (In,Ga)As reference and the two (In, Ga,Mn)As films.

Magnetic properties and the Curie temperature $\left(T_{\mathrm{C}}\right)$ values for the (In,Ga,Mn)As films were determined using both magnetic-field- and temperature-dependent SQUID magnetometry. Customary cut long $\mathrm{Si}$ strips facilitate sample support in the magnetometer chamber [39]. All the data presented here have their relevant diamagnetic contributions of the substrate evaluated at room temperature and subtracted adequately.

\section{Combination of bulk valence-band mapping and photoelectron diffraction in one experiment}

A key element of the present approach is the possibility to switch between full-field imaging HARPES, yielding the valence-band dispersions in the bulk, and hXPD, yielding structural information. The experiment has been performed at beamline P22 [40] of the Synchrotron source Petra III (DESY, Hamburg), belonging to the hard-x-ray beamlines with the highest brilliance worldwide. The instrument has been described in detail in [41]. The three-dimensional (3D) recording architecture (full-field $k_{\mathrm{x}}-k_{\mathrm{y}}$ imaging combined with time-of-flight energy recording) overcomes the intensity/resolution problem of HARPES. Moreover, full-field imaging allows the removal of the strong hXPD-modulations in valence-band $k$-distributions [42]. A multiplicative correction is applied using core-level hXPD patterns recorded at the same kinetic energy that eliminates the modulations [34]. Hard-X-ray $k$-microscopy allows tomographic imaging of the $4 \mathrm{D}$ spectral-density function $\rho\left(\mathrm{E}_{\mathrm{B}}, \boldsymbol{k}\right)$, tuning the third momentum coordinate $k_{\mathrm{z}}$ by variation of the photon energy [35].

A simplified view of the instrument is shown in the Supplemental Material, Fig. S1 [43]. The achromatic momentum image (reciprocal image formed in the back-focal plane of the lens) is zoomed to the desired size by a lens stack and recorded by a delay-line detector (DLD). A low-energy drift section serves as an energy-dispersing element. The measurements were performed with the characteristic parameters: $k$-field diameter $\sim 10 \AA^{-1}, k$-resolution down to $0.025 \AA^{-1}\left(0.034^{\circ}\right.$ angular resolution), kinetic energy up to $7 \mathrm{keV}$, time-of-flight energy resolution $\sim 40 \mathrm{meV}$ (measured at $h v=6 \mathrm{keV}$ photon energy), and photon bandwidth $330 \mathrm{meV}$ (for $3300 \mathrm{eV}$ ) for the $\mathrm{Si}(111)$ monochromator crystal; for details, see Ref. [41].

\section{RESULTS AND DISCUSSION}

\section{A. Hole concentrations}

The results of micro-Raman scattering spectroscopy reveals that the longitudinal-optical (LO) phonon mode couples with the hole-gas-related plasmon, forming the so-called coupled plasmon-LO-phonon mode (CPPM) for (In,Ga,Mn)As. However, we do not observe this feature in the spectra of 


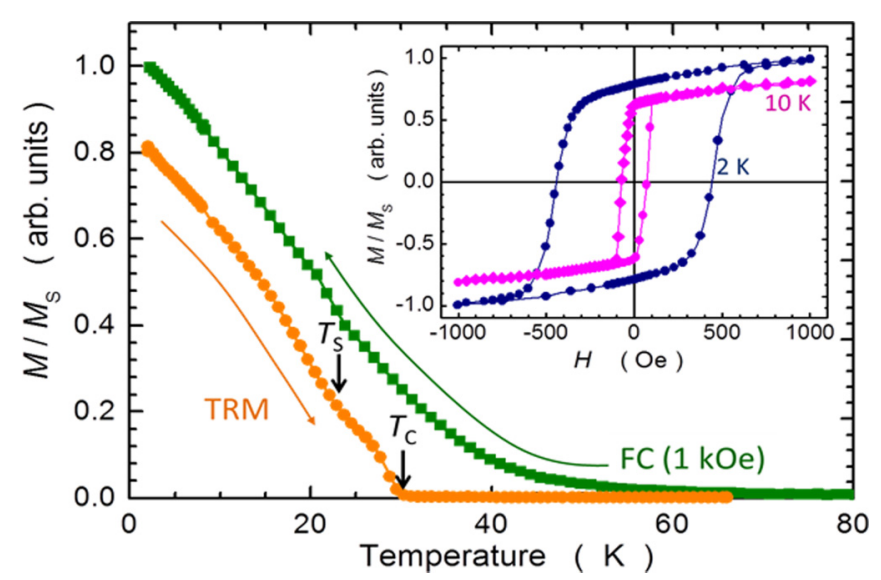

FIG. 1. Main panel: Temperature dependence of the magnetization $M$ in an (In,Ga,Mn)As film with $5.6 \% \mathrm{Mn}$. The sample is initially field-cooled (FC) in a magnetic field of $H=1 \mathrm{kOe}$ (squares), and after quenching the field the thermoremanent magnetization (TRM) is recorded during warming (circles). The temperature at which TRM vanishes indicates the Curie temperature $\left(T_{\mathrm{C}}\right) . T_{\mathrm{S}}$ indicates the spin reorientation transition between uniaxial (above $T_{\mathrm{S}}$ ) and biaxial (below $T_{\mathrm{S}}$ ) in-plane magnetic anisotropies [48]. Inset: Hysteresis curves at 2 and $10 \mathrm{~K}$. All measurements are performed with $H$ applied in-plane along the $[-110]$ uniaxial easy axis. $M$ is expressed with respect to the saturation value $M_{\mathrm{S}}=M(2 \mathrm{~K}, 1 \mathrm{kOe})$.

the reference (In, Ga)As layers. In this case, only a strong LO-phonon line and a very weak (symmetry-forbidden) transverse-optical (TO) phonon line located around 290 and $260 \mathrm{~cm}^{-1}$, respectively, are observed.

From micro-Raman spectra, the hole concentrations have been estimated to be about $10^{19}$ and $10^{20} \mathrm{~cm}^{-3}$ in the films with $2.5 \%$ and $5.6 \%$ Mn content, respectively $[44,45]$. The obtained results suggest that, similarly to $(\mathrm{Ga}, \mathrm{Mn}) \mathrm{As}$ [44], the (In,Ga,Mn)As film with $2.5 \% \mathrm{Mn}$ shows properties of an insulator-like material whereas the film with $5.6 \% \mathrm{Mn}$ is similar to a metallic one [46].

It is well known that an As excess-mainly in the form of arsenic antisites, $\mathrm{As}_{\mathrm{Ga}}$ - occurs at low-temperature $\mathrm{MBE}$ growth conditions. $\mathrm{As}_{\mathrm{Ga}}$ antisites act as deep double donors and lead to an $n$-type hopping conductivity in LT-GaAs. Similarly, very diluted (In,Ga,Mn)As is $n$-type, too. The threshold Mn concentration between $n$ - and $p$-type conduction depends on the actual concentration of $\mathrm{As}_{\mathrm{Ga}}$ donors and therefore on the actual MBE chamber set up and the growth conditions [47]. With further increase of Mn concentration, the CPPM mode starts to dominate the spectra, simultaneously with its energy shifted toward the TO-phonon line. This is the direct indication of an increasing hole density with increasing $x$, which can be quantified from the full line-shape fitting [46].

\section{B. Magnetic properties}

Figure 1 reveals the ferromagnetic behavior with a nonvanishing remnant magnetization and open hysteresis loops $M(H)$ of the $x=5.6 \%$ (In, Ga,Mn)As film at low temperatures. Further measurements (not shown here) reveal an in-plane [-110] easy axis, a characteristic feature ubiquitously met in $(\mathrm{Ga}, \mathrm{Mn})$ As films [48]. We determine $T_{\mathrm{C}}$ in this sample from the temperature dependence of the remnant magnetization (TRM) measured along this easy direction. The established magnitude of $T_{\mathrm{C}}=30 \mathrm{~K}$ is typical for nonannealed $(\mathrm{Ga}, \mathrm{Mn})$ As films [24]. A small inflection point at around $T_{S} \cong 23 \mathrm{~K}$ and a rounded shape of both $M(H)$ loops (both are measured below $T_{S}$ ) indicate a spin reorientation transition due to a change from the in-plane uniaxial to an in-plane biaxial magnetic anisotropy, taking place at $T_{S}$ [49]. SQUID magnetometry has revealed a nonferromagnetic character of the $2.5 \% \mathrm{Mn}$-doped (In,Ga,Mn)As layers. It is understood that due to the smaller $x$ the free hole concentration is too low to induce a long-range ferromagnetic order above $2 \mathrm{~K}$. This suggestion is supported by the micro-Raman measurements, which reveal that the hole concentration in this layer is below $10^{19} \mathrm{~cm}^{-3}$.

\section{Recording hXPD signatures of Ga and As sites exploiting the noncentrosymmetry of (In,Ga,Mn)As}

Photoelectron diffraction patterns have been recorded at final-state energies of $\sim 3.31$ and $5.11 \mathrm{keV}$, and the results are shown in Figs. 2 and 3, respectively. The final-state energy is the kinetic energy of the photoelectrons in the crystal (see [35]), being the relevant quantity for the diffraction dynamics. To have exactly the same kinetic energies and identical momentum scales for the Ga and As diffractograms, the photon energies are tuned to the values given in the figure captions.

The patterns consist of a pronounced system of Kikuchi bands and lines with a rich fine structure. The experimental and theoretical diffractograms show a one-to-one agreement of the overall structure, the widths of the observed bands, and the positions of the lines. Differences occur only in the relative intensities. Eye-catching features are the vertical and horizontal Kikuchi bands of $\{110\}$-type, with their bright crossing region in the center. The width of this band is given by the reciprocal lattice vector $\left|G_{110}\right|=3.14 \AA^{-1}$. Another general feature is the system of dark lines throughout the entire pattern. The diameter of the field of view for $5.11 \mathrm{keV}$ (Fig. 3) is $16 \AA^{-1}$, corresponding to a polar angular range of only $+/-13^{\circ}$. All patterns exhibit a horizontal and a vertical mirror plane, parallel to the [110] and [1-10] directions.

Figure 2 shows the results for the final-state energy of $3.31 \mathrm{keV}$. Close inspection of the Ga (a), (b) and As (c), (d) diffractograms reveals systematic differences, both in the measured and calculated patterns. Arrows with numbers mark characteristic features. As eye-catching signatures, we find two arcs (1) in dark gray, running from left to right for $\mathrm{Ga}$ and from top to bottom for As $\left(1^{\prime}\right)$. These arcs (1) and (1') appear in the calculations $(b, d)$ in the same orientations as in the experiment, i.e., rotated by $90^{\circ}$ with respect to each other. This is a clear hint on the different orientation of the coordination tetrahedron; see the sketch in Fig. 2(g). A second fingerprint are bright horseshoe-shaped features $\left(2,2^{\prime}\right)$ appearing in the four diagonal directions, which touch the arcs close to the rim of the field-of-view. The horseshoes are oriented close to horizontal for Ga (a), (b) and close to vertical for As (c), (d). Another characteristic are the faint dark crosses $\left(3,3^{\prime}\right)$ in the center of the diffractograms (a)-(d). The larger angle $>90^{\circ}$ (marked) is on top (bottom) for Ga (a), (b) and the left (right) side for As (c), (d). We find an excellent agreement 


\section{Ga 3d}
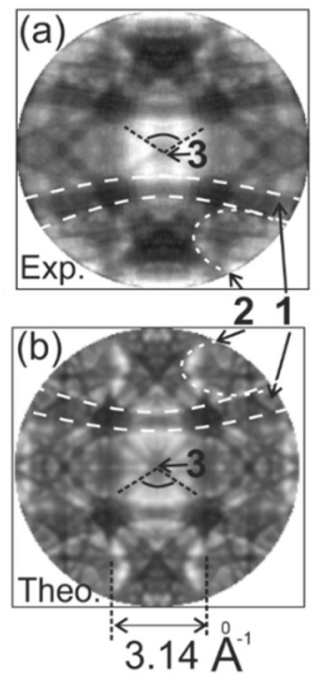

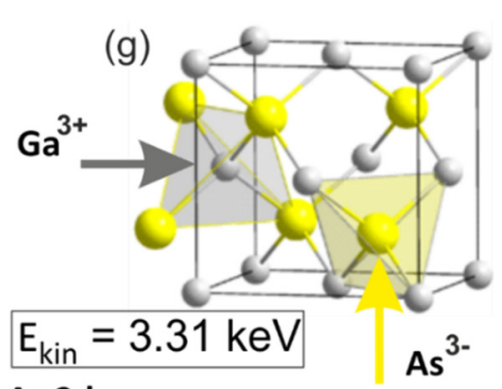

As 3d

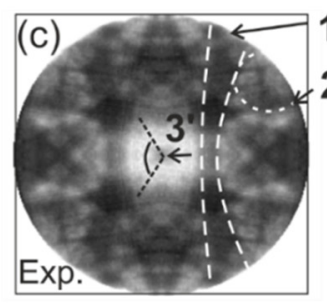

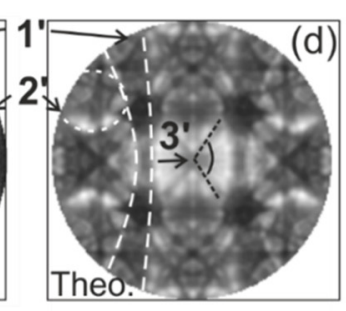

As 3d/Ga 3d

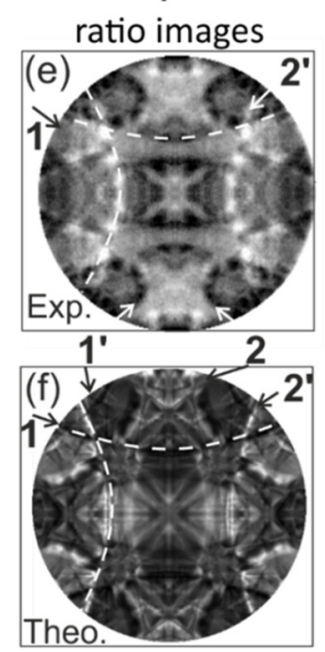

FIG. 2. Measured (a), (c) and calculated (b), (d) diffraction patterns of photoelectrons from the Ga $3 d_{5 / 2}$ and As $3 d_{5 / 2}$ core levels at a kinetic energy of $3.31 \mathrm{keV}$. Data were recorded for a GaAs (001) thin film doped with $3 \% \mathrm{In}$ and $2.5 \% \mathrm{Mn}$; experimental patterns are twofold symmetrized. Identical $E_{\text {kin }}$ was set by tuning the photon energy to $3317 \mathrm{eV}$ for Ga (a) and to $3340 \mathrm{eV}$ for As (c). (e), (f) Ratio images of the As- and Ga-diffractograms, emphasizing the differences originating from the missing inversion center of the zinc-blende-type lattice. (g) GaAs unit cell with indicated tetrahedral coordination of the $\mathrm{Ga}^{3+}$ and $\mathrm{As}^{3-}$ ions. Note that all $\mathrm{Ga}$ - and As-tetrahedra are rotated by $90^{\circ}$ with respect to each other.

between experiment and theory. The lower resolution in the experimental patterns might indicate a smaller experimental coherence length due to a smaller inelastic mean free path than assumed for the calculation (cf. Fig. 3 in [50]). Further differences show up in relative intensities, as was found in our previous experiment on graphite (cf. Fig. 5 in [49]). These measurements have been performed for the In- and Mn-doped samples.

The differences in the diffractograms for As and Ga emitter atoms can be emphasized by means of ratio images, derived via pixel-by-pixel division of the As (c) and Ga patterns (a). Experimental and theoretical ratio images are shown in (e)
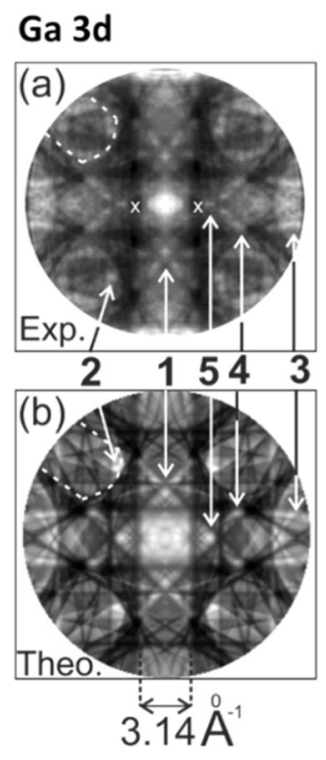

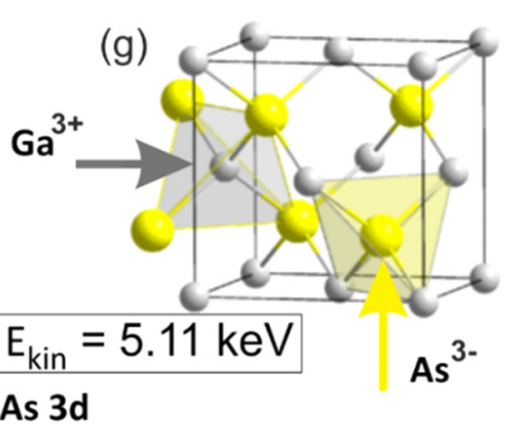

As 3d

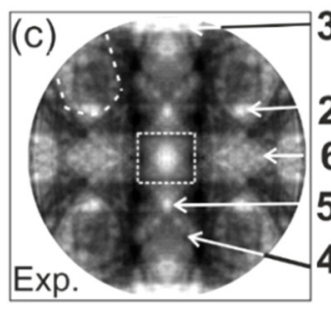

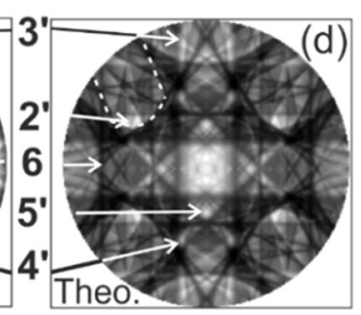

As 3d/Ga 3d
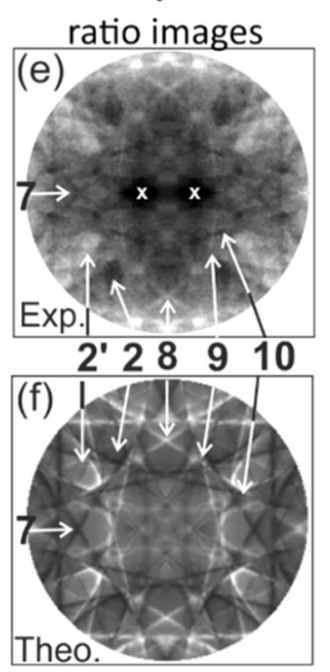

FIG. 3. Measured (a), (c) and calculated (b), (d) Ga $3 d_{5 / 2}$ and As $3 d_{5 / 2}$ core-level photoelectron diffraction patterns at a kinetic energy of $5.11 \mathrm{keV}$. (e), (f) Measured and calculated ratio images of the As- and Ga-diffractograms. Like Fig. 2 for a GaAs (001) thin film doped with $3 \%$ In and $2.5 \% \mathrm{Mn}$, but for a kinetic energy of $5.11 \mathrm{keV}$, set by tuning the photon energy to $5117 \mathrm{eV}$ for Ga $3 d$ (a) and to $5140 \mathrm{eV}$ for As (c). The inner part of pattern (c), marked by a square, is shown with different gray level in order to avoid intensity saturation. $\times$ marks small dark detector artefacts. 

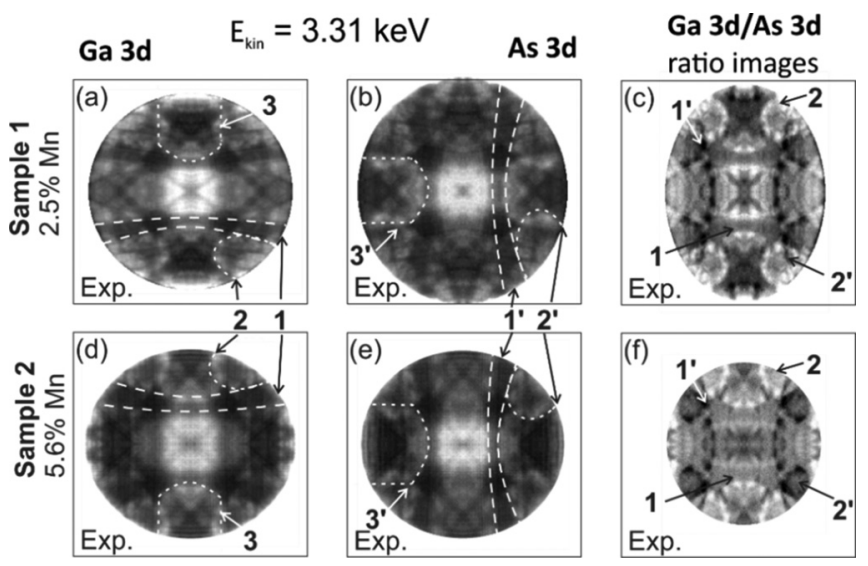

FIG. 4. Measured Ga $3 d_{5 / 2}$ and As $3 d_{5 / 2}$ core-level photoelectron diffractograms and corresponding ratio images for sample 1 with $2.5 \% \mathrm{Mn}(\mathrm{a})-(\mathrm{c})$ and sample 2 with $5.6 \% \mathrm{Mn}(\mathrm{d})-(\mathrm{f})$ for a kinetic energy of $3.31 \mathrm{keV}$. In the ratio images, outer regions with significant distortions have been cropped, leading to the elliptical shape.

and (f), respectively. Indeed, the characteristic differences are strongly enhanced. In particular, the horseshoe features show up very pronounced in bright (2) and dark dotted lines $\left(2^{\prime}\right)$, dark because the As pattern is in the denominator. Analogously, the arcs appear as rows of bright $\left(1^{\prime}\right)$ and dark dots (1) that originate in corresponding rows of dots at the outer side of the arcs in (a)-(d). Experimental (e) and theoretical ratio images (f) agree very well in all main features. Differences in details are mostly due to the lower resolution of the experiment because of pixel noise and different relative intensities.

Figure 3 shows the analogous results for the final-state energy of $5.11 \mathrm{keV}$. Due to the different photoelectron wavelength, the patterns are markedly different from those in Fig. 2. In the Ga-diffractograms (a), (b) we recognize sixfold stars (1) above and below the center, bright spots (2) in four horseshoe-shaped features along the diagonal directions, four bright stripes (3) at the left and right rims, and two V-shaped structures (4) with bright spots at their tips (5) left and right of the center. The As-diffractograms (c,d) show four bright spots $\left(2^{\prime}\right)$ in differently oriented horseshoes, four bright stripes $\left(3^{\prime}\right)$ at the upper and lower rim, and two V-shaped patterns $\left(4^{\prime}\right)$ with bright spots $\left(5^{\prime}\right)$ above and below the center. As for $3.31 \mathrm{keV}$, all As features appear rotated by $90^{\circ}$ in comparison with Ga. In Fig. 5(c), the sixfold stars are not present; instead, we observe a diamond-shaped field with inner fine structure (6). This diamond feature appears in the calculation (d) as well, but its inner fine structure looks somewhat different.

Figures 3(e) and 3(f) show the As $3 d / \mathrm{Ga} 3 d$ ratio images. The experimental ratio image (e) shows a rich fine structure of narrow bright and dark lines, which agree perfectly well with the calculated ratio image (f). Note, e.g., the dark (7) and bright crosses (8), as well as the tilted bright (9) and dark lines $(10)$. Crosses $(7,8)$ and tilted lines $(9,10)$ are again rotated by $90^{\circ}$ with respect to each other. The bright spots (2) in (a) and $\left(2^{\prime}\right)$ in (c) appear as four characteristic dark-bright features $\left(2,2^{\prime}\right)$ in (e) and (f).

Ratio images like those in Figs. 3(e) and 4(e) offer a simple way to eliminate experimental artefacts. Inhomogeneities such as uneven illumination of the field of view, effects of distortions due to the electron optics, or local sensitivity variations of the detector cancel, because these are present in both images (a), (c). Hence, many fine details are emphasized, exploiting the high resolution of the microscope. The comparison of Figs. 4(e) and 4(f) demonstrates again the one-to-one correspondence of all Kikuchi lines. Actually, this result is not surprising because it is analogous to a Laue diffraction pattern, where the spot positions are solely determined by the reciprocal lattice. Here, each Kikuchi line represents a reciprocal-lattice vector (corresponding to a set of lattice planes). Laue spots and Kikuchi lines are both manifestations of the metric of the reciprocal lattice.

Figure 4 shows a comparison of results taken at identical final-state energies of $3.31 \mathrm{keV}$ and identical settings of the microscope for two samples with different Mn content, $2.5 \%$ (top row) and 5.6\% (bottom row). Comparison of the diffractograms (a), (b), (d), (e) and ratio images (c), (f) shows that all signatures discussed in Fig. 2 are present for both dopant contents. In addition, we have marked the large horseshoes $\left(3,3^{\prime}\right)$ with a central cross and a dark triangle. The pair of horseshoes is oriented vertically for Ga and horizontally for As.

In the ratio images (c), (f), arc 1 from the Ga patterns shows up as a chain of bright spots, and arc $1^{\prime}$ from the As patterns appears as a chain of dark spots. Analogously, the horseshoes 2 and $2^{\prime}$ appear bright and dark in (c), (f). The elliptical outer shape results from cropping of distorted regions. The patterns in the second row appear slightly more blurred in comparison with the first row. This can be attributed to a higher degree of disorder due to the larger Mn content. Moreover, the central regions in the ratio images (c), (f) show significant differences. This may point to increased lattice distortions with increasing Mn content. Distortions on a larger scale in real space show up in the central region in reciprocal space.

\section{Analysis of the sites of the dopants in the host lattice}

The previous section has shown that the Ga and As sites in the GaAs lattice have fingerprint-like signatures of their Kikuchi patterns. The missing inversion symmetry allows an unambiguous assignment of substitutional sites, provided the kinetic energy of the core-level electrons of the dopant is tuned to exactly the same value as used for the reference diffractograms [51]. The question is, however, whether concentrations in the percent range provide a sufficient signal for an assignment. The results can be compared with previous work dealing with the location of Mn sites [52-55].

Figure 5 shows the diffraction patterns of the four constituents measured at the final-state energy of $3.31 \mathrm{keV}$. Dotted lines and numbers mark characteristic features. Inspection of the Mn $3 d$ hXPD pattern (c) reveals that the arc (1) runs from left to right, and the horseshoe with a center cross is visible on the top and bottom. This is the signature of the Ga substitutional site, in agreement with previous work. Due to the small signal-to-noise ratio of the core-level spectrum, contrast and resolution are reduced in comparison with the main constituents. The pattern had to be smoothed in order to remove statistical noise. The In $3 d$ pattern shows only a very weak contrast, which is not sufficient for an unambiguous assignment of the Ga site. 


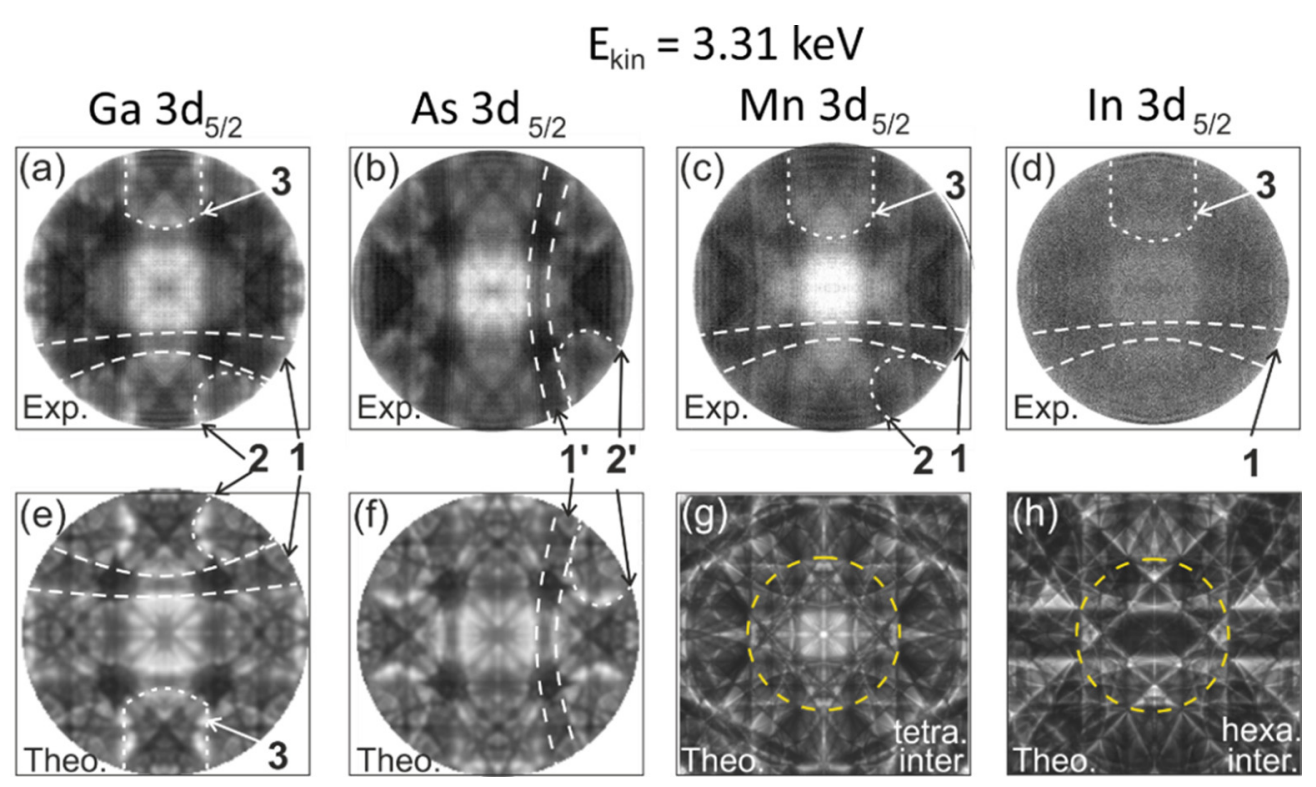

FIG. 5. (a)-(d) Hard-x-ray diffraction patterns of photoelectrons from Ga $3 d_{5 / 2}$, As $3 d_{5 / 2}$, Mn $3 d_{5 / 2}$, In $3 d_{5 / 2}$ of the (In,Ga,Mn)As thin film with nominal doping of $3 \%$ and $5.6 \%$ of $\mathrm{In}$ and $\mathrm{Mn}$, respectively. The kinetic energy is set to $3.31 \mathrm{keV}$ by tuning the photon energies to $\mathrm{h} v=3317,3340,3355$, and $3342 \mathrm{eV}$ for (a), (b), (c), and (d). (e)-(h) Kikuchi diffraction calculation for Mn atoms on Ga (e) and As sites (f) as well as for Mn atoms on tetrahedral (g) and hexagonal interstitial sites (h), all for $E_{\mathrm{kin}}=3.31 \mathrm{keV}$. Dotted lines and numbers mark characteristic features; the dashed circles in $(\mathrm{g})$, (h) denote the field-of-view of (a)-(f).

The second row of Fig. 5 shows calculated Kikuchi diffractograms for $\mathrm{Mn}$ atoms on $\mathrm{Ga}(\mathrm{e})$ and As sites (f), which are identical to the $\mathrm{Ga}$ and As patterns at the same final-state energy. The signatures are in accordance with the experimental patterns (a), (b). They show the same orientation of arcs (1) for $\mathrm{Ga}$ and $\left(1^{\prime}\right)$ for As, of the small horseshoes along the diagonal $\left(2,2^{\prime}\right)$, and of the larger horseshoes with a cross $\left(3,3^{\prime}\right)$. Incorporation of $\mathrm{Mn}$ on the substitutional $\mathrm{Ga}$ sites is also evident from the comparison of (c) and (e).

Since they have been discussed as possible Mn sites, we also calculated the Kikuchi fingerprints of $\mathrm{Mn}$ atoms on tetrahedral and hexagonal interstitial sites. Mn on interstitial sites in the GaAs lattice has been analyzed theoretically by Blinowski and Kacman [56] and Mahadevan and Zunger [51]. Indications for such sites have been found by NEXAFS [57], Rutherford backscattering [52], and electron spin resonance (ESR) [58].

Along with the two types of interstitials with tetragonal and hexagonal symmetry in the zinc-blende lattice, there are two nonequivalent tetrahedral interstitial sites with different Kikuchi patterns. The tetrahedral interstitials can be coordinated to either four As or four Ga atoms. If the dopant atoms would occupy energetically identical sites statistically, the observed Kikuchi patterns would be the average of the two equivalent sites. Calculations revealed, however, that the tetrahedral interstitial site coordinated by As is more stable than that coordinated by Ga. This would induce a dissymmetry in the occupation number. For this reason, we have calculated the Kikuchi fingerprints of both types of interstitial sites separately. A detailed discussion of the site-specificity of Kikuchi patterns is given in [50].

The calculated hXPD patterns of Mn on tetrahedral and hexagonal interstitial sites are shown in Figs. 5(g) and 5(h), respectively. The hexagonal site is characterized by dark diagonal Kikuchi bands of $\{001\}$-type that cross the horizontal $\{011\}$-band. The crossing region appears very dark. This is not compatible with the bright center region of the measured diffractogram (c). The tetrahedral interstitial (g) shows a bright center region but the rest of the pattern does not resemble the experimental pattern (c). For instance, the four large dark spots at the corners of the central bright rectangle in (c) are a prominent feature in the calculation for the Ga site (e) but are very weak in the interstitial calculation (g). From the comparison of experimental and calculated patterns, we conclude that Mn predominantly occupies substitutional $\mathrm{Ga}$ sites, even in the hyperdoping regime.

The impressive agreement of Bloch-wave theory with experiment in Figs. 2, 3, and 4 as well as for graphite [49] and $\mathrm{Si}$ [50] bears an important message. For such materials with low scattering factors [59], Bragg reflection at sets of lattice planes is a fast and effective model to analyze and interpret hXPD patterns. The computational efficiency of the $k$-space description by the Bloch-wave approach lies in the small number $(\sim 100)$ of Fourier components (corresponding to sets of lattice planes) that need to be considered. It is clear, though, that real-space (i.e., cluster-type) calculations in principle yield identical results if fully converged, as proven by comparative calculations in [60]. Modeling a detail-rich Kikuchi-pattern like those in Figs. 2 and 3 in a cluster-type calculation would demand including $\sim 10^{6}$ coherent scatterers, which constitutes a major computational challenge. On the other hand, cluster calculations do not assume long-range periodicity, which is crucial for Kikuchi diffraction. Hence, cluster codes are sensitive to the local arrangement close to the emitter atom and can easily calculate adsorbates on the surface.

To summarize, the hXPD analysis of the site of the $\mathrm{Mn}$ atoms, being responsible for the ferromagnetism of 

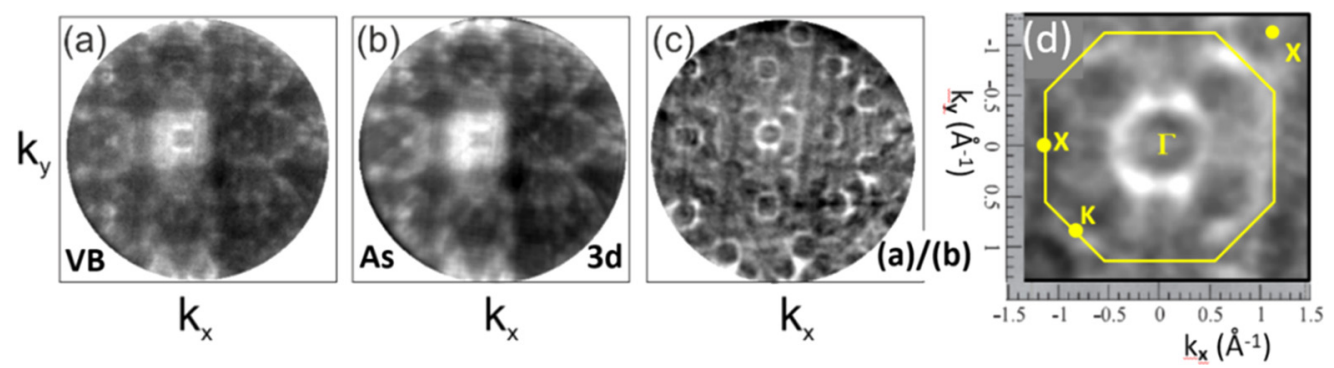

FIG. 6. Momentum distributions $I\left(k_{\mathrm{x}}, k_{y}\right)$ of photoelectrons from (In,Ga,Mn)As (001) measured at a kinetic energy of $3.3 \mathrm{keV}(T=25 \mathrm{~K})$. (a) Constant-energy section of the as-measured valence-band distribution at a binding energy of $E_{\mathrm{B}}=1 \mathrm{eV}$, dominated by the Kikuchi pattern signature originating from valence-band photoelectron diffraction. (b) Kikuchi pattern of photoelectrons from the As $3 d$ core-level. (c) Valenceband distribution with diffraction eliminated by multiplicative correction, i.e., pixel-by-pixel division of patterns (a) and (b). Now the bulk valence bands in $\sim 12$ Brillouin zones (BZs), arranged in a square array, are clearly visible. (d) Close-up view of one BZ with marked zone boundary and high-symmetry points $\Gamma, X$, and $K$.

(In,Ga,Mn)As, was performed for the low- and high-doping regime. In the high-doping regime, the influence of the Mn content on the band structure is still an open question, and the dopant site in the host lattice is discussed intensely and controversially. Due to its noncentrosymmetric structure, the zinc-blende lattice of GaAs offers the possibility to distinguish the Ga and As sites via an hXPD analysis. In conventional Laue diffraction and Kikuchi diffraction (e.g., in a scanning electron microscope), the missing inversion center is invisible due to Friedel's law. Prior to the present work, it was unclear whether core-level photoelectron Kikuchi diffraction would make the missing inversion center visible. These site-specific Kikuchi patterns at identical kinetic energies are used as reference fingerprints for substitutional dopant sites. Tetrahedral and hexagonal interstitial sites are identified employing simulated Kikuchi patterns, exploiting their proven one-to-one correspondence with experiment $[49,50]$.

\section{E. Influence of Mn concentration on the bulk spectral function}

Band mapping with hard $\mathrm{x}$-rays is hampered by a strong contribution of valence-band photoelectron diffraction [34,42], which can be largely eliminated by multiplicative correction using a core-level diffraction pattern recorded at the identical kinetic energy. Thanks to time-of-flight recording, single counting events are accumulated in $\mathrm{I}\left(E_{\mathrm{B}}, k_{\mathrm{x}}, k_{y}\right)$ histogram arrays. Such arrays allow a posteriori analysis of $k_{\mathrm{x}}-k_{\mathrm{y}}$ sections at any desired energy or $E_{\mathrm{B}}-k_{\|}$sections in any direction in $k$-space. Since the Debye-Waller factor is relevant at such high energies, the patterns have been recorded at a sample temperature of $25 \mathrm{~K}$. Figure 6 shows what this diffraction correction looks like for the title compound. The as-measured valence-band pattern (a) looks very similar to the As $3 d$ core-level diffractogram (b). Note that (a) shows one energy from a 3D data array of several $\mathrm{eV}$ width. The energy resolution is defined by the photon bandwidth of $330 \mathrm{meV}$ at this photon energy $(3.3 \mathrm{keV})$.

In the pixel-by-pixel ratio image (c), the intensity modulation by the Kikuchi pattern is eliminated, uncovering the true valence-band features. The $k$-field-of-view comprises many $(\sim 12)$ Brillouin zones (BZs) in a square array, originating from the section of the final-state energy isosphere with the periodic pattern of [001]-oriented BZs comprising all band features in 3D $k$-space at the given final-state energy. In valence-band photoelectron diffraction, the final-state energy (here $3.3 \mathrm{keV}$ ) governs the diffraction dynamics because it defines the photoelectron wavelength. The binding energy (here $1 \mathrm{eV}$ ) is not less important, because it defines on which energy isosurface (one of which is the Fermi surface) the diffraction condition is fulfilled; for details, see [42]. Figure 6(d) shows a detail of the band features, the central $\mathrm{BZ}$ with the contour of the BZ boundary and the high-symmetry points $\Gamma, X$, and $K$ being marked. The pattern is dominated by an intense band centered around the $\Gamma$-point, a weaker band close to $\Gamma$, and bright features at the periphery of the pattern in the diagonal $\Gamma-K$ directions.

The $k$-space metric is visible both in the width of the Kikuchi bands in (a), (b) and in the distance of equivalent points of the band structure in (c). Given the lattice constant of $a=5.6533 \AA$, the width of the band is $\left|G_{110}\right|=3.14 \AA^{-1}$, and the distance between $\Gamma$ and $X$ along the image diagonal is $\left|G_{110}\right| / 2 \sqrt{ } 2=1.11 \AA^{-1}$.

Figure 7 shows the photoemission distribution for the photon energy of $3300 \mathrm{eV}$. This energy has been chosen to cut the three-dimensional Brillouin zone in the $\Gamma-X-K$ plane [Fig. 7(a)], assuming a free-electron final-state model. Previous work has shown that this approximation is valid at such high energies [35,41]. Variation of the photon energy shows that the photoemission intensity at $E_{F}$ and at the $\Gamma$ point is highest at $3.3 \mathrm{keV}$, confirming the probed section of momentum-space.

In Fig. 7(b), energy-dispersive spectra measured in the vicinity of the $\Gamma$-point are displayed for $(\mathrm{In}, \mathrm{Ga}) \mathrm{As}$ with $2.5 \%$ and $5.6 \% \mathrm{Mn}$ doping. The spectral weight at $E_{F}$ increases with increasing Mn doping, which is in agreement with previous results $[61-63,21]$. The position of the chemical potential is referenced to a metallic $\mathrm{Co}_{2} \mathrm{MnSi}$ sample measured prior to the present measurements with identical settings. For the 5.6\% Mn-doped sample, no band dispersion is visible above this energy. Therefore, this energy level represents an upper limit for $E_{F}$. For both Mn concentrations of 2.5\% and 5.6\% one observes the valence-band dispersions of the light-hole (LH) and split-off band (SO) for both high symmetry directions $\Gamma-X$ and $\Gamma-K$ as shown in Figs. 7(c)-7(f). The heavy-hole (HH) band is barely visible at these high energies, in agreement with [21]. Figures 7(g)-7(r) display the intensity distributions 
(a)
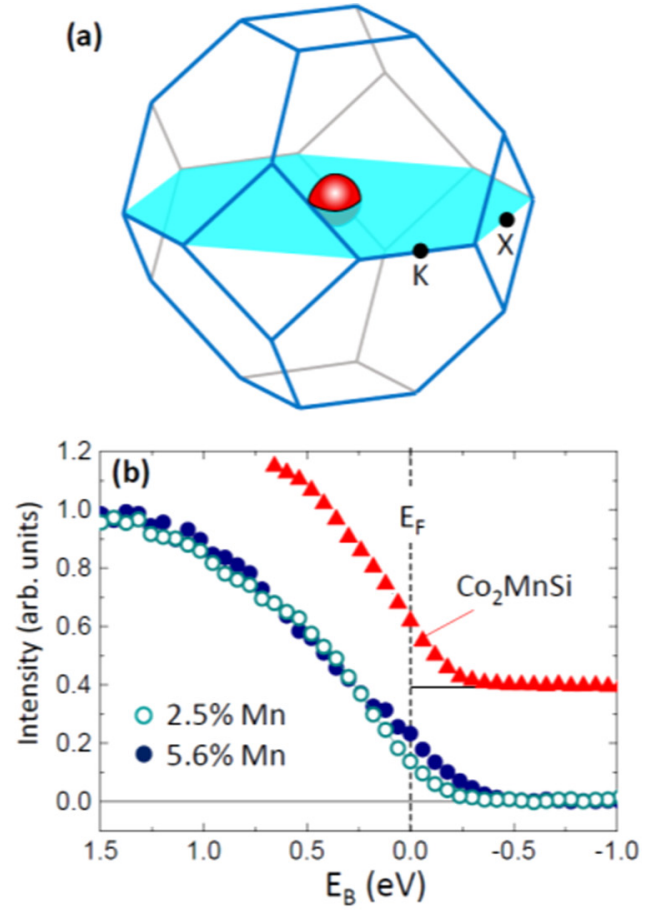
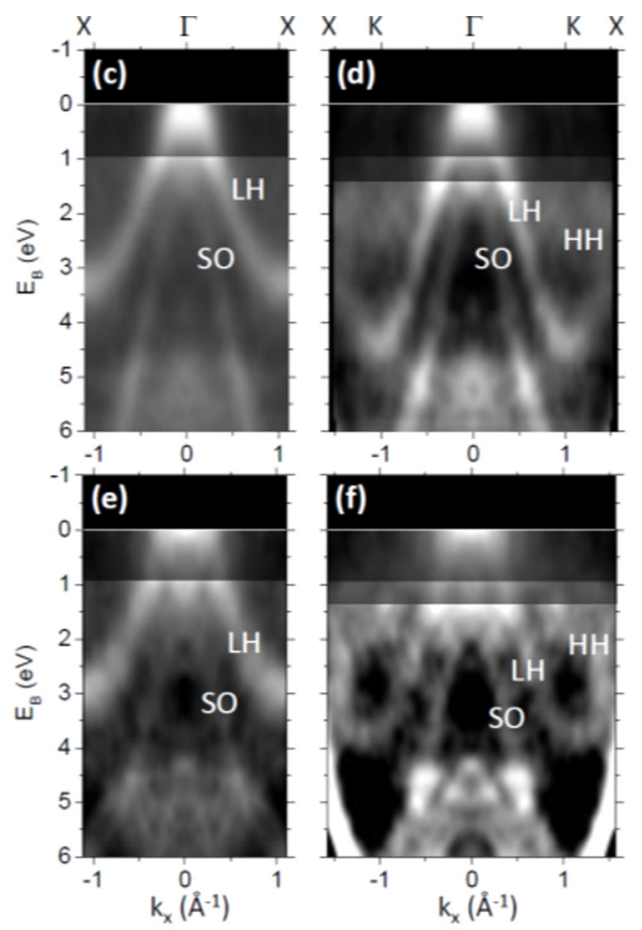
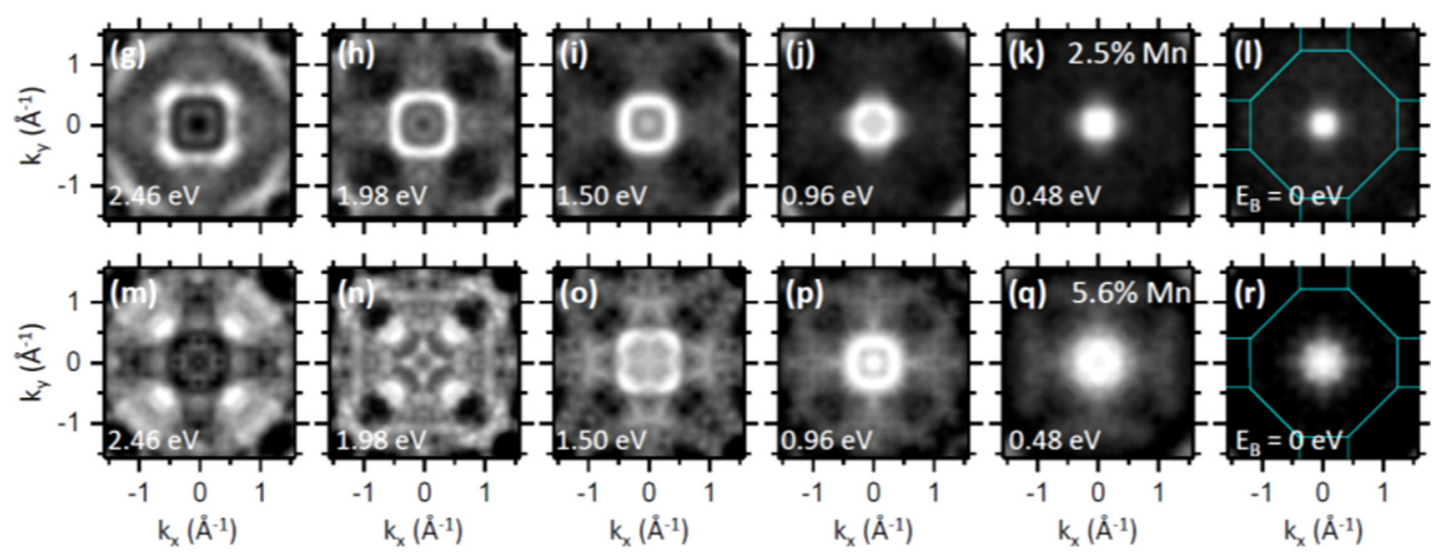

FIG. 7. Valence-band dispersion of Mn-doped (In,Ga)As. (a) Bulk Brillouin zone of (In,Ga)As with the indicated $\Gamma$ - $X$ - $K$ plane probed by momentum microscopy with a photon energy of $3.300 \mathrm{eV}$. (b) Comparison of raw ARPES spectra around the $\Gamma$ point [integrated over a circle with diameter $0.2 \AA^{-1}$ centered at the $\Gamma$ point, red sphere in (a)] for (In,Ga,Mn)As thin films with nominal doping of $2.5 \%$ and $5.6 \%$ Mn and for a metallic $\mathrm{Co}_{2} \mathrm{MnSi}$ reference sample. The intensity is normalized at $E_{\mathrm{B}}=1.5 \mathrm{eV}$. (c), (d) $E_{\mathrm{B}}$ vs $k_{\|}$along the $\Gamma-X$ (c) and $\Gamma-K$ direction (d) for $2.5 \% \mathrm{Mn}$-doping. The displayed photoemission intensity distribution is normalized to the average intensity of a constant energy $k_{\mathrm{x}}-k_{\mathrm{y}}$ map and the background is subtracted. (e), (f) Similar data for Mn doping of 5.6\%; the horizontal stripes in the top regions of (c)-(f) are shown with different contrast to enhance the dynamic range. Parts $(\mathrm{g})-(\mathrm{l})$ show the corresponding $k_{\mathrm{x}}-k_{\mathrm{y}}$ sections at selected binding energies for $2.5 \%$ Mn doping. (m)-(r) Similar data for Mn doping of 5.6\%. HH, LH, and SO denote the heavy-hole, light-hole, and split-off bands, respectively.

as constant energy plots for both samples as a function of in-plane wave vector at selected energy slices revealing the dispersion of the LH and SO bands. The constant energy contours of the LH and SO bands show a circular shape for binding energies smaller than $1 \mathrm{eV}$. For larger binding energy, the LH band becomes fourfold distorted, whereas the SO band retains its circular shape.

Figure 8 shows a comparison of the experimental band dispersions with the tight-binding calculation for pristine GaAs at the $\Gamma-X-K$ plane taken from Ref. [60]. The position of the $\mathrm{LH}$ and $\mathrm{SO}$ bands in the experimental $E_{\mathrm{B}}$ versus $k_{\|}$data has been determined from the maxima in the momentum distribution curves (minima in the second derivative). We rigidly shifted the calculated data on the energy scale to fit the experimental data. In the case of $2.5 \%$ Mn doping, the experimental data are compatible with the valence-band maximum positioned at $E_{F}$ [Fig. 8(a)]. For $5.6 \%$ Mn doping, we observe the dispersing LH band up to the Fermi level, and the valence-band maximum lies above $E_{F}$. Using the tight-binding model of Ref. [60] for extrapolation, the valence-band maximum lies $250 \mathrm{meV}$ above the Fermi level. The spin-orbit-induced energy splitting of the split-off band appears larger than the value of $\Delta E_{\mathrm{SO}}=$ $0.34 \mathrm{eV}$ for pristine GaAs [64]. This can be partly explained 


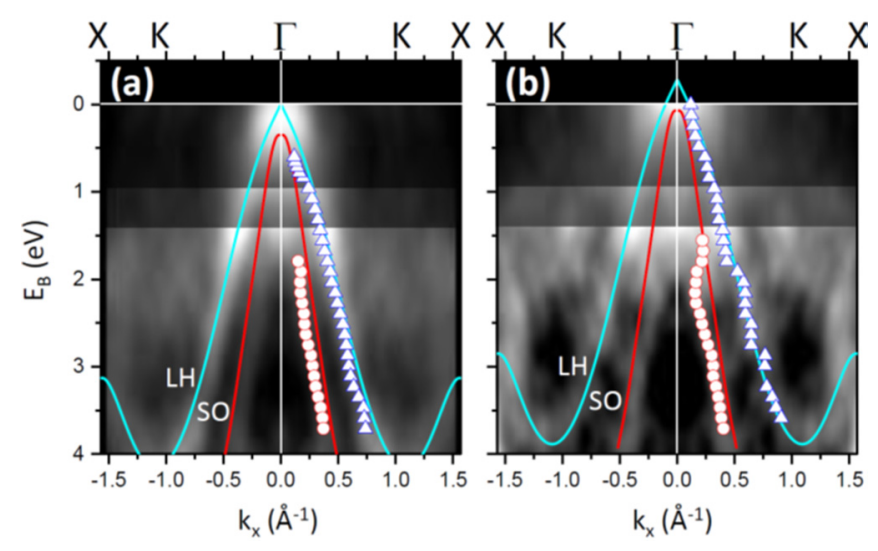

FIG. 8. Valence-band dispersion of Mn-doped (In,Ga)As for $2.5 \%$ (a) and $5.6 \%$ (b) Mn doping. The red open circles (blue open triangles) indicate minima of the second derivative of momentum distribution curves for the split-off band SO (light-hole band LH). The red (light blue) solid lines represent a tight-binding calculation for pristine GaAs (taken from Ref. [60]), rigidly shifted to match the experimental LH positions.

by the In content. For InAs, a value of $\Delta E_{\mathrm{SO}}=0.4 \mathrm{eV}$ has been reported [63]. Within the binding energy range from 2 to $3 \mathrm{eV}$, the $\mathrm{SO}$ band shows a deviation from the linear dispersion calculated by the tight-binding method that increases with increasing Mn doping level.

To disclose less obvious band deformations, Fig. 9(a) shows a pair of intensity profiles along the $\Gamma-K-X$ direction at selected binding energies. We chose the energies such that the in-plane momenta of the LH band $\left(\Delta k_{\mathrm{LH}}\right)$ are the same for both Mn dopings. Then, the in-plane momentum of the SO band $\left(\Delta k_{\mathrm{SO}}\right)$ for $5.6 \%$ doping is clearly smaller than in the case of $2.5 \%$ doping. Figure 9(b) reveals how this difference in parallel momentum develops along the bands in the binding energy range 1.5-3.5 eV. At larger momentum values (larger binding energy), both datasets show a linear change of $\Delta k_{\mathrm{SO}}$ with $\Delta k_{\mathrm{LH}} . \Delta k_{\mathrm{SO}}$ is smaller for the $5.6 \% \mathrm{Mn}$ doping by $0.1 \AA^{-1}$ with respect to $2.5 \%$ doping. This smaller momentum translates into a rigid shift of the SO band with respect to the LH band to higher binding energy by $0.8 \mathrm{eV}$. For smaller parallel momentum (smaller binding energy), the two datasets merge within error limits. A similar plot reported for Mn-doped GaAs [22] reveals a smaller difference between GaAs and Mn-doped (3\%) GaAs, with $\Delta k_{\text {So }}$ being slightly larger for the Mn-doped case in the lower binding energy range. Thus, Fig. 9 shows that the Mn-doping modifies the bulk band structure of pristine (InGa)As in a nontrivial way.

In classical models, the split-off energy results from a combination of spin-orbit coupling and crystal field. At the $\Gamma$-point, the band states are often described in an atomiclike picture, where the $\mathrm{HH}$ - and $\mathrm{LH}$-states correspond to hole states with quantum numbers $p_{3 / 2}\left|m_{\mathrm{j}}\right|=3 / 2$ and $p_{3 / 2}\left|m_{\mathrm{j}}\right|=$ $1 / 2$, respectively. These two states are split by the crystal field. In this picture, the SO state corresponds to a $p_{1 / 2}$ hole state, which is spherically symmetric. The observed shift with increasing Mn content thus cannot be explained in a single electron picture, because the Mn does not increase the spinorbit interaction nor does it substantially change the crystal
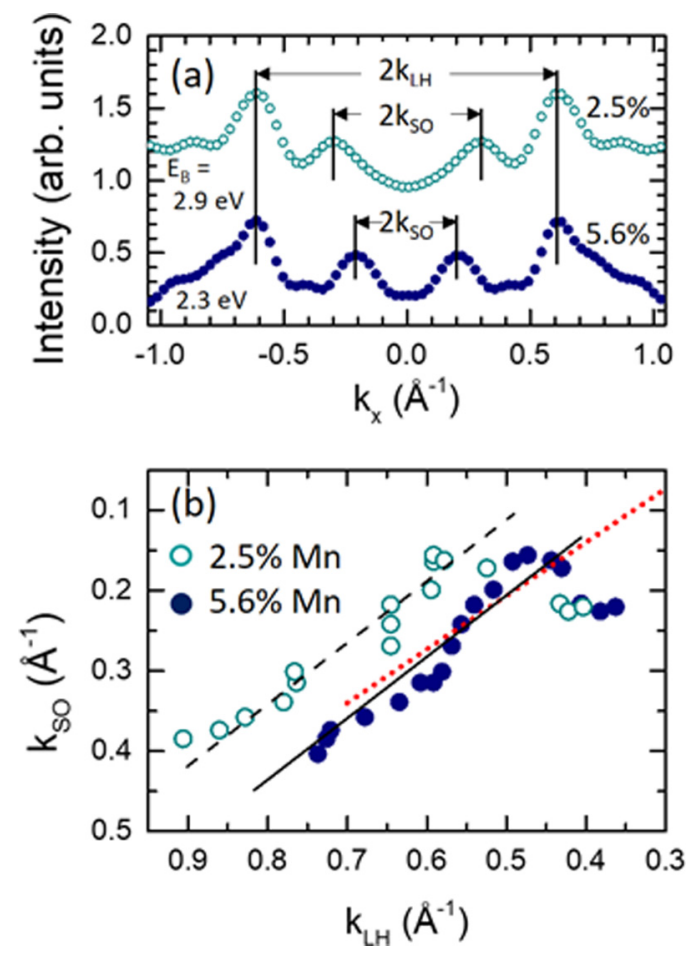

FIG. 9. (a) Intensity profiles extracted at the indicated binding energy values for (In, Ga)As doped with $2.5 \%$ and $5.6 \% \mathrm{Mn}$, respectively. (b) The parallel momentum of the SO band $\left(k_{\mathrm{SO}}\right)$ as a function of the corresponding momentum of the $\mathrm{LH}$ band $\left(k_{\mathrm{LH}}\right)$ for the two samples. The black dashed and full lines are linear fits to the data for $k_{\mathrm{LH}}<0.6 \AA^{-1}(2.5 \%)$ and $k_{\mathrm{LH}}<0.55 \AA^{-1}(5.6 \%)$. The red dotted line represents the tight-binding result.

field. The partial replacement of $\mathrm{Ga}$ with In increases the average nuclear charge $Z$ and hence increases the split-off energy with respect to pure GaAs [63]. The replacement of Ga with $\mathrm{Mn}$ instead lowers the average $Z$-value, and one would have expected a reduced split-off energy with increasing $\mathrm{Mn}$ content, in contrast to our experimental observation. Hence, tight-binding or effective potential models cannot explain the origin of the band modifications.

In the following, we discuss the Mn-induced changes of the band structure. Thanks to the high kinetic energy of $\sim 3.3 \mathrm{keV}$, the experiment probes the bulk band structure of the samples with an information depth of about $10 \mathrm{~nm}$. The contribution of the surface region to the total signal is negligible, thus avoiding surface-related effects like the interface band bending caused by surface impurity states. For the high carrier concentrations of the samples investigated here, and considering the low temperature of the measurement, the space-charge region can extend only 1-2 $\mathrm{nm}$ into the bulk of the sample. This information is important for the following discussion of the band positions.

$\mathrm{Mn}^{2+}$ ions on $\mathrm{Ga}$ sites act as strong acceptors, because their acceptor states are more localized than the hydrogeniclike acceptor states in p-doped GaAs (e.g., Zn-doped). The more localized character results in higher critical carrier density of the metal-insulator transition in Mn-doped GaAs $\left(\sim 10^{20} \mathrm{~cm}^{-3}\right)$. For $\mathrm{Mn}$ concentrations beyond the metalinsulator transition $(\sim 1.6 \%)$, the Mn acceptor levels define 
the position of the Fermi level, which then shifts below the valence-band maximum.

The observed band structure remains largely persistent even for high Mn concentrations, indicating the presence of mobile holes residing in a nearly unperturbed valence band of the GaAs host. These mobile holes are essential for the $p-d$ Zener model of ferromagnetism discussed for diluted magnetic semiconductors. In contrast, the alternatively discussed model for ferromagnetism considers localized Mn impurity states above the GaAs Fermi level, carrying a localized magnetic moment that couples via indirect exchange interaction with the conduction holes. The latter model requires that the Fermi level stays fixed at the valence-band maximum. This scenario can be excluded by the present measurements, in agreement with previous work [21,22].

We can directly compare our experimental results with recent work. Reference [60] reports ARPES data measured with $21 \mathrm{eV}$ excitation for GaAs doped with 3\% Mn. The photoemission intensity decreases near the chemical potential, not allowing for a direct detection of the valence-band maximum. Comparing their data with a tight-binding model for pure GaAs, the authors extrapolate that the Fermi level crosses the valence band below its maximum in the case of ferromagnetic Mn-doped GaAs. An alternative interpretation of the same ARPES data has been given in Refs. $[19,20]$ arguing that the impurity band model with the valence-band maximum below the chemical potential would also be consistent with the data. Reference [22], using $25 \mathrm{eV}$ synchrotron radiation, found that Mn concentrations above $1 \%$ induce a highly dispersive band that reaches the Fermi level and extends into the gap region of GaAs, i.e., its maximum lies above the valence-band maximum. Yet, the highly dispersive band does not explain the high charge-carrier density found in DFS, and the authors conclude that localized Mn states are formed in parallel with the highly dispersive band. This debate is partly related to the surface sensitivity of the above-mentioned experiments, requiring the assumption that bulk properties are detected.

In our case, the high kinetic energy suppresses photoemission yield from surface states or any other surface-related band modifications. For the case of the ferromagnetic sample $(5.6 \% \mathrm{Mn})$, we observe the dispersive LH valence band up to the chemical potential with a finite parallel momentum of $0.1 \AA^{-1}$. This finding cannot be interpreted with a picture where all dispersing bands lie below the chemical potential. Our experimental data are in qualitative agreement with those of Refs. $[60,22]$. A quantitative agreement cannot be expected since we investigated a different host material. The limited energy resolution does not allow us to distinguish the subtle difference of whether the dispersive band crossing the chemical potential is Mn-induced or Mn-derived. Yet, we do observe a fuzzy broadening and high intensity of the photoemission signal at the chemical potential, which would be compatible with the occurrence of additional localized Mn states in this region.

In the case of the SO band, we find a particularly strong band modification in the binding energy range between 2 and $3 \mathrm{eV}$ along the [110] in-plane direction $(\Gamma-K-X)$. This finding is in rough agreement with the spectral function calculated by many-body theory [dynamical mean field theory (DMFT)] [21] for the case of Mn-doped GaAs. This might be taken as a hint that the observed band modifications with respect to, e.g., tight-binding calculations require many-body theory, including the effects of the dopant-induced hole gas [22].

\section{SUMMARY AND CONCLUSIONS}

The investigation of the tailoring of the electronic structure in diluted magnetic semiconductors in analogy to conventional semiconductors represents an experimental challenge, because local structural order may vary and conventional ARPES with low photon energy excitation probes only the topmost atomic layers. Therefore, we applied hXPD and HARPES to demonstrate the conservation of the broken inversion symmetry and the band structure tailoring for highquality MBE-grown (In, Ga,Mn)As films with $3 \%$ In plus $2.5 \%$ or $5.6 \% \mathrm{Mn}$. Hard-x-ray excitation provides the necessary bulk sensitivity to avoid surface-induced band structure corruptions. HARPES and hXPD probe identical sample volumes and identical depth profiles, thus ensuring that the probed volume is the same for the geometric and electronic information.

For the determination of the site-specific atomic order using hXPD, selected core levels of the constituents are addressed via tuning of the photon energy. Simulated hXPD patterns using the Bloch-wave method show a one-to-one correspondence with the experimental results and allow us to predict the specific signatures of different dopant sites. For samples with $2.5 \%$ and 5.6\% Mn content, the Kikuchi patterns confirm that $\mathrm{Mn}$ predominantly occupies substitutional $\mathrm{Ga}$ sites.

The bulk electronic structure of the (In,Ga,Mn)As films results from the spectral function $I\left(E_{\mathrm{B}}, k_{\mathrm{x}}, k_{\mathrm{y}}\right)$ in the valenceband region as measured using HARPES. HARPES reveals that a strongly dispersing band crosses the Fermi energy in the highly doped sample. This is a consequence of the high concentration of Mn-induced itinerant holes residing in the valence band of the III-V semiconductor host. This result is not in agreement with a pure impurity band model. In agreement with previous studies, we find an increased density of states near the Fermi level for higher doping levels, which one may attribute to additional localized Mn states. In addition to a shift of the chemical potential with increasing Mn-doping, we find pronounced band modifications, particularly in the case of the split-off band.

\section{ACKNOWLEDGMENTS}

Sincere thanks are due to Christoph Schlueter, Juriy Matveyev, and Andrii Gloskovskii (DESY, Hamburg) for excellent support during the beamtimes at beamline P22, PETRA III. We gratefully acknowledge financial support by BMBF (Projects No. 05K19UM1 and No. 05K19UM2) and by Deutsche Forschungsgemeinschaft (Transregio SFB 173 Spin $+X$ 268565370, projects A02 and A05). J.S. acknowledges the support from the Swedish Research Council (VR) within Project No. 2017-04404. O.Y. and M.S. are thankful to the National Academy of Science of Ukraine and Polish Academy of Science for bilateral cooperation grant. A.W. has been supported by the Polish National Agency for Academic Exchange (NAWA) Grant No. PPN/ULM/2019/1/00068/U/00001. 
[1] T. Dietl and H. Ohno, Dilute ferromagnetic semiconductors: Physics and spintronic structures, Rev. Mod. Phys. 86, 187 (2014).

[2] H. Ohno, A. Shen, F. Matsukura, A. Oiwa, A. Endo, S. Katsumoto, and Y. Iye, (Ga,Mn)As: A new diluted magnetic semiconductor based on GaAs, Appl. Phys. Lett. 69, 363 (1996)

[3] M. Wang, R. P. Campion, A. W. Rushforth, K. W. Edmonds, C. T. Foxon, and B. L. Gallagher, Magnetic coupling in ferromagnetic semiconductor (Ga,Mn)As/(Al,Ga,Mn)As bilayers, Appl Phys. Lett. 93, 132103 (2008).

[4] L. Chen, X. Yang, F. Yang, J. Zhao, J. Misuraca, P. Xiong, and S. von Molnár, Enhancing the Curie temperature of ferromagnetic semiconductor (Ga,Mn)As to $200 \mathrm{~K}$ via nanostructure engineering, Nano Lett. 11, 2584 (2011).

[5] E. Rozkotova, P. Nemec, P. Horodyska, D. Sprinzl, F. Trojanek, P. Maly, V. Novak, K. Olejnik, M. Cukr, and T. Jungwirth, Direct measurement of the three-dimensional magnetization vector trajectory in GaMnAs by a magneto-optical pump-andprobe method, Appl. Phys. Lett. 92, 122507 (2008).

[6] M. Sawicki, F. Matsukura, A. Idziaszek, T. Dietl, G. M. Schott, C. Ruester, C. Gould, G. Karczewski, G. Schmidt, and L. W. Molenkamp, Temperature dependent magnetic anisotropy in (Ga,Mn)As layers, Phys. Rev. B 70, 245325 (2004).

[7] D. Chiba, M. Sawicki, Y. Nishitani, Y. Nakatani, F. Matsukura, and $\mathrm{H}$. Ohno, Magnetization vector manipulation by electric fields, Nature (London) 455, 515 (2008).

[8] N. Gonzalez Szwacki, J. A. Majewski, and T. Dietl, (Ga,Mn)As under pressure: A first-principles investigation. Phys. Rev. B 91, 184409 (2015).

[9] M. Gryglas-Borysiewicz, A. Kwiatkowski, P. Juszyński, Z. Ogorzałek, K. Puźniak, M. Tokarczyk, G. Kowalski, M. Baj, D. Wasik, N. G. Szwacki, J. Przybytek, J. Sadowski, M. Sawicki, P. Dziawa, and J. Z. Domagala, Hydrostatic pressure influence on TC in (Ga,Mn)As, Phys. Rev. B 101, 054413 (2020).

[10] L. Li, S. D. Yao, S. Zhou, D. Bürger, O. Roshchupkina, S. Akhmadaliev, A. W. Rushforth, R. P. Campion, J. Fassbender, and M. Helm, Tailoring the magnetism of GaMnAs films by ion irradiation, J. Phys. D 44, 045001 (2011).

[11] T. Jungwirth, J. Wunderlich, V. Novák, K. Olejník, B. L. Gallagher, R. P. Campion, K. W. Edmonds, A. W. Rushforth, A. J. Ferguson, and P. Němec, Spin-dependent phenomena and device concepts explored in (Ga,Mn)As, Rev. Mod. Phys. 86, 855 (2014).

[12] S. Das Sarma, E. H. Hwang, and A. Kaminski, How to make semiconductors ferromagnetic: a first course on spintronics, Solid State Commun. 127, 99 (2003).

[13] T. Jungwirth, J. Sinova, J. Mašek, J. Kučera, and A. H. MacDonald, Theory of ferromagnetic (III,Mn)V semiconductors, Rev. Mod. Phys. 78, 809 (2006).

[14] F. Máca, J. Kudrnovský, O. Makarovsky, L. Eaves, R. P. Campion, K. W. Edmonds, A. W. Rushforth, C. T. Foxon, B. L. Gallagher, V. Novák, J. Sinova, and T. Jungwirth, Microscopic Analysis of the Valence Band and Impurity Band Theories of (Ga,Mn)As, Phys. Rev. Lett. 105, 227202 (2010).

[15] G. Bouzerar and R. Bouzerar, Unraveling the nature of carriermediated ferromagnetism in diluted magnetic semiconductors (Elucider la nature du ferromagnétisme induit par des porteurs dans les semiconducteurs magnétiques dilués), C. R. Phys. 16, 731 (2015).
[16] T. Dietl, H. Ohno, F. Matsukura, J. Cibert, and D. Ferrand, Zener model description of ferromagnetism in zinc-blende magnetic semiconductors, Science 287, 1019 (2000).

[17] T. Jungwirth, J. Sinova, A. H. MacDonald, B. L. Gallagher, V. Novák, K. W. Edmonds, A. W. Rushforth, R. P. Campion, C. T. Foxon, L. Eaves, E. Olejník, J. Mašek, S.-R. E. Yang, J. Wunderlich, C. Gould, L. W. Molenkamp, T. Dietl, and H. Ohno, Character of states near the Fermi level in (Ga,Mn)As: Impurity to valence band crossover, Phys. Rev. B 76, 125206 (2007).

[18] K. Alberi, K. M. Yu, P. R. Stone, O. D. Dubon, W. Walukiewicz, T. Wojtowicz, X. Liu, and J. K. Furdyna, Formation of Mn-derived impurity band in III-Mn-V alloys by valence band anticrossing, Phys. Rev. B 78, 075201 (2008).

[19] M. Kobayashi, S. Ohya, I. Muneta, Y. Takeda, Y. Harada, J. Krempasky, T. Schmitt, M. Oshima, V. N. Strocov, M. Tanaka, and A. Fujimori, Alternative interpretation of the recent experimental results of angle-resolved photoemission spectroscopy on GaMnAs, arXiv:1608.07718.

[20] M. Kobayashi, I. Muneta, Y. Takeda, Y. Harada, A. Fujimori, J. Krempasky, T. Schmitt, S. Ohya, M. Tanaka, M. Oshima, and V. N. Strocov, Unveiling the impurity band inducing ferromagnetism in magnetic semiconductor (Ga,Mn)As, Phys. Rev. B 89, 205204 (2014).

[21] A. X. Gray, J. Minár, S. Ueda, P. R. Stone, Y. Yamashita, J. Fujii, J. Braun, L. Plucinski, C. M. Schneider, G. Panaccione, H. Ebert, O. D. Dubon, K. Kobayashi, and C. S. Fadley, Bulk electronic structure of the dilute magnetic semiconductor $\mathrm{Ga}(1-\mathrm{x}) \mathrm{Mn}(\mathrm{x})$ As through hard X-ray angle-resolved photoemission, Nat. Mater. 11, 957 (2012).

[22] J. Kanski, L. Ilver, K. Karlsson, I. Ulfat, M. Leandersson, J. Sadowski, and I. Di Marco, Electronic structure of (Ga,Mn)As revisited, New. J. Phys. 19, 023006 (2017).

[23] T. Dietl, A. Bonanni, and H. Ohno, Families of magnetic semiconductors-An overview, J. Semicond. 40, 080301 (2019).

[24] O. Yastrubchak, J. Sadowski, L. Gluba, J. Z. Domagala, M. Rawski, J. Żuk, M. Kulik, T. Andrearczyk, and T. Wosinski, Ferromagnetism and the electronic band structure in (Ga,Mn)(Bi,As) epitaxial layers, Appl. Phys. Lett. 105, 072402 (2014).

[25] K. Levchenko, T. Prokscha, J. Sadowski, I. Radelytskyi, R. Jakiela, M. Trzyna, T. Andrearczyk, T. Figielski, and T. Wosinski, Evidence for the homogeneous ferromagnetic phase in $(\mathrm{Ga}, \mathrm{Mn})(\mathrm{Bi}, \mathrm{As})$ epitaxial layers from muon spin relaxation spectroscopy, Sci. Rep. 9, 3394 (2019).

[26] S. Ohya, H. Kobayashi, and M. Tanaka, Magnetic properties of heavily Mn-doped quaternary alloy ferromagnetic semiconductor (InGaMn)As grown on InP, Appl. Phys. Lett. 83, 2175 (2003).

[27] K. Levchenko, T. Andrearczyk, J. Z. Domagala, J. Sadowski, L. Kowalczyk, M. Szot, R. Kuna, T. Figielki, and T. Wosiński, Novel quaternary dilute magnetic semiconductor $(\mathrm{Ga}, \mathrm{Mn})(\mathrm{Bi}, \mathrm{As})$ : Magnetic and magneto-transport investigations, J. Supercond. Nov. Magn. 30, 825 (2017).

[28] Im. T. Yoon, S. Lee, Y. Shon, Y. Kwon, Y. S. Park, and T. W. Kang, Optical and magnetic properties of ten-period InGaMnAs/GaAs quantum wells, J. Supercond. Nov. Magn. 26, 3529 (2013). 
[29] Y. H. Kwon, S. Lee, W. Yang, C.-S. Park, and Im T. Yoon, Ferromagnetic properties of five-period InGaMnAs/GaAs quantum well structure, J. Electron. Mater. 46, 3917 (2017).

[30] J. Wang, J. Kono, A. Oiwa, H. Munekata, and C. J. Stanton, Ultrafast carrier dynamics in ferromagnetic InGaMnAs, Superlattices Microstruct. 34, 563 (2003).

[31] J. Sadowski, R. Mathieu, P. Svedlindh, J. Kanski, M. Karlsteen, K. Swiatek, and J. Domagala, Magnetic properties of short period InGaMnAs/InGaAs superlattices, Acta. Phys. Pol. A 102, 687 (2002).

[32] T. Dietl, H. Ohno, and F. Matsukura, Hole-mediated ferromagnetism in tetrahedrally coordinated semiconductors, Phys. Rev. B 63, 195205 (2001).

[33] T. Slupinski, H. Munekata, and A. Oiwa, Ferromagnetic semiconductor (In, Ga,Mn)As with Curie temperature above $100 \mathrm{~K}$, Appl. Phys. Lett. 80, 1592 (2002).

[34] S. Babenkov, K. Medjanik, D. Vasilyev, S. Chernov, C. Schlueter, G. Gloskovskii, Yu. Matveyev, W. Drube, B. Schönhense, K. Rossnagel, H.-J. Elmers, and G. Schönhense, High-accuracy bulk electronic bandmapping with eliminated diffraction effects using hard X-ray photoelectron momentum microscopy, Commun. Phys. 2, 107 (2019).

[35] K. Medjanik, O. Fedchenko, S. Chernov, D. Kutnyakhov, M. Ellguth, A. Oelsner, B. Schönhense, T. R. F. Peixoto, P. Lutz, C.-H. Min, F. Reinert, F. Däster, Y. Acremann, J. Viefhaus, W. Wurth, H.-J. Elmers, and G. Schönhense, Direct 3D mapping of the fermi surface and fermi velocity, Nat. Mater. 16, 615 (2017).

[36] O. Yastrubchak, J. Żuk, H. Krzyżanowska, J. Z. Domagala, T. Andrearczyk, J. Sadowski, and T. Wosinski, Photoreflectance study of the fundamental optical properties of (Ga,Mn)As epitaxial films, Phys. Rev. B 83, 245201 (2011).

[37] O. Yastrubchak, J. Bak-Misiuk, E. Łusakowska, J. Kaniewski, J. Z. Domagala, T. Wosiński, A. Shalimov, K. Regiński, and A. Kudla, Strain release in InGaAs/InxAl1-xAs/InP heterostructures, Physica B 340-342, 1082 (2003).

[38] J. Sadowski, J. Z. Domagala, J. Bąk-Misiuk, S. Koleśnik, M. Sawicki, K. Świątek, J. Kanski, L. Ilver, and V. Ström, Structural and magnetic properties of molecular beam epitaxy grown GaMnAs layers, J. Vac. Sci. Technol. B 18, 1697 (2000).

[39] M. Sawicki, W. Stefanowicz, and A. Ney, Sensitive SQUID magnetometry for studying nano-magnetism, Semicond. Sci. Technol. 26, 064006 (2011).

[40] C. Schlueter, A. Gloskovskii, K. Ederer, S. Piec, M. Sing, R. Claessen, C. Wiemann, C.-M. Schneider, K. Medjanik, G. Schönhense, P. Amann, A. Nilsson, and W. Drube, The New Dedicated HAXPES Beamline P22 at PETRAIII, AIP Conf. Proc. No. 2054 (AIP, New York, 2019), p. 040010.

[41] K. Medjanik, S. Babenkov, S. Chernov, D. Vasilyev, B. Schönhense, C. Schlueter, A. Gloskowskii, Yu. Matveyev, W. Drube, H. J. Elmers, and G. Schönhense, Progress in HAXPES performance combining full-field k-imaging with time-of-flight recording, J. Synch. Radiat. 26, 1996 (2019).

[42] G. Schönhense, K. Medjanik, S. Babenkov, D. Vasilyev, M. Ellguth, O. Fedchenko, S. Chernov, B. Schönhense, and H.-J. Elmers, Momentum-transfer model of valence-band photoelectron diffraction, Commun. Phys. 3, 45 (2020).

[43] See Supplemental Material at http://link.aps.org/supplemental/ 10.1103/PhysRevB.103.075107 for a simplified view of the instrument.
[44] L. Gluba, O. Yastrubchak, J. Z. Domagala, R. Jakiela, T. Andrearczyk, J. Żuk, T. Wosinski, J. Sadowski, and M. Sawicki, Band structure evolution and the origin of magnetism in Ga,Mn)As: From paramagnetic through superparamagnetic to ferromagnetic phase, Phys. Rev. B 97, 115201 (2018).

[45] Y. Yuan, C. Xu, R. Hübner, R. Jakiela, R. Böttger, M. Helm, M. Sawicki, T. Dietl, and S. Zhou, Interplay between localization and magnetism in (Ga,Mn)As and (In,Mn)As, Phys. Rev. Mater. 1, 054401 (2017).

[46] F. Matsukura, H. Ohno, A. Shen, and Y. Sugawara, Transport properties and origin of ferromagnetism in $(\mathrm{Ga}, \mathrm{Mn}) \mathrm{As}$, Phys. Rev. B 57, R2037 (1998).

[47] O. Yastrubchak, J. Sadowski, H. Krzyzanowska, L. Gluba, J. Zuk, J. Z. Domagala, T. Andrearczyk, and T. Wosinski, Electronic- and band-structure evolution in low-doped (Ga,Mn)As, J. Appl. Phys. 114, 053710 (2013).

[48] M. Sawicki, Magnetic properties of (Ga,Mn)As, J. Magn. Magn. Mater. 300, 1 (2006).

[49] M. Sawicki, K.-Y. Wang, K. W. Edmonds, R. P. Campion, C. R. Staddon, N. R. S. Farley, C. T. Foxon, E. Papis, E. Kamińska, A. Piotrowska, T. Dietl, and B. L. Gallagher, In-plane uniaxial anisotropy rotations in (Ga,Mn)As thin films, Phys. Rev. B 71, 121302(R) (2005).

[50] O. Fedchenko, A. Winkelmann, K. Medjanik, S. Babenkov, D. Vasilyev, S. Chernov, C. Schlueter, A. Gloskowskii, Yu. Matveyev, W. Drube, B. Schönhense, H. J. Elmers, and G. Schönhense, High-resolution hard-X-ray photoelectron diffraction in a momentum microscope-The model case of graphite, New. J. Phys. 21, 113031 (2019).

[51] O. Fedchenko, A. Winkelmann, S. Chernov, K. Medjanik, S. Babenkov, S. Agustsson, D. Vasilyev, M. Hoesch, H.-J. Elmers, and G. Schönhense, Emitter-site specificity of hard X-ray photoelectron Kikuchi-diffraction, New J. Phys. 22, 103002 (2020).

[52] P. Mahadevan and A. Zunger, Ferromagnetism in Mn-doped GaAs due to substitutional-interstitial complexes, Phys. Rev. B 68, 075202 (2003).

[53] K. M. Yu, W. Walukiewicz, T. Wojtowicz, I. Kuryliszyn, X. Liu, Y. Sasaki, and J. K. Furdyna, Effect of the location of Mn sites in ferromagnetic $\mathrm{Ga}_{1-x} \mathrm{Mn}_{x}$ As on its Curie temperature, Phys. Rev. B 65, 201303(R) (2002).

[54] B. Grandidier, J. P. Nys, C. Delerue, and D. Stiévenard, Atomicscale study of GaMnAs/GaAs layers, Appl. Phys. Lett. 77, 4001 (2000).

[55] J. Sadowski and J. Z. Domagala, Influence of defects on the lattice constant of GaMnAs, Phys. Rev. B 69, 075206 (2004).

[56] J. Blinowski and P. Kacman, Spin interactions of interstitial Mn ions in ferromagnetic GaMnAs, Phys. Rev. B 67, 121204(R) (2003).

[57] R. Shioda, K. Ando, T. Hayashi, and M. Tanaka, Local structures of III-V diluted magnetic semiconductors $\mathrm{Ga}_{1-x} \mathrm{Mn}_{x} \mathrm{As}$ studied using extended x-ray-absorption fine structure, Phys. Rev. B 58, 1100 (1998).

[58] S. J. C. H. M. van Gisbergen, M. Godlewski, T. Gregorkiewicz, and C. A. J. Ammerlaan, Magnetic-resonance studies of interstitial Mn in GaP and GaAs, Phys. Rev. B 44, 3012 (1991).

[59] L.-M. Peng, Electron atomic scattering factors and scattering potentials of crystals, Micron 30, 625 (1999).

[60] A. Winkelmann, C. S. Fadley, and F. J. Garcia de Abajo, Highenergy photoelectron diffraction: Model calculations and future possibilities, New J. Phys. 10, 113002 (2008). 
[61] S. Souma, L. Chen, R. Oszwałdowski, T. Sato, F. Matsukura, T. Dietl, H. Ohno, and T. Takahashi, Fermi level position, Coulomb gap and Dresselhaus splitting in $(\mathrm{Ga}, \mathrm{Mn}) \mathrm{As}$, Sci. Rep. 6, 27266 (2016).

[62] J. Fujii, B. R. Salles, M. Sperl, S. Ueda, M. Kobata, K. Kobayashi, Y. Yamashita, P. Torelli, M. Utz, C. S. Fadley, A. X. Gray, J. Braun, H. Ebert, I. Di Marco, O. Eriksson, P. Thunstrom, G. H. Fecher, H. Stryhanyuk, E. Ikenaga, J. Minar, C. H. Back, G. van der Laan, and G. Panaccione, Identifying the
Electronic Character and Role of the Mn States in the Valence Band of (Ga,Mn)As, Phys. Rev. Lett. 111, 097201 (2013).

[63] J. Okabayashi, A. Kimura, O. Rader, T. Mizokawa, A. Fujimori, T. Hayashi, and M. Tanaka, Angle-resolved photoemission study ofGa ${ }_{1-x} \mathrm{Mn}_{x}$ As, Phys. Rev. B 64, 125304 (2001).

[64] I. Vurgaftman, J. R. Meyer, and L. R. Ram-Mohan, Band parameters for III-V compound semiconductors and their alloys, J. Appl. Phys. 89, 5815 (2001). 